\title{
Triglyceride to high-density lipoprotein cholesterol ratio is associated with increased mortality in older patients on peritoneal dialysis
}

\author{
Xiaojiang Zhan ${ }^{\dagger}$, Mei Yang ${ }^{\dagger}$, Ruitong Zhou, Xin Wei, Yanbing Chen and Qinkai Chen ${ }^{*}$
}

\begin{abstract}
Background: The triglyceride ( $T G$ ) to high-density lipoprotein cholesterol (HDL-C) ratio (TG/HDL-C) has been suggested as a simple method to identify unfavorable cardiovascular (CV) outcomes in the general population. The aim of this study was to investigate the association between the TG/HDL-C ratio and all-cause and CV mortality in peritoneal dialysis (PD) patients.
\end{abstract}

Methods: We retrospectively analyzed patients on PD from November 1, 2005, to February 28, 2017, with a follow-up period lasting until May 31, 2017. The main outcomes were all-cause and CV mortality.

Results: Among the 973 PD patients, the mean age was $49.67 \pm 14.58$ (y). During a median follow-up period of 27.2 months (IQR = 13.4-41.5 months), 229 (23.5\%) patients died, with 120 (12.3\%) dying as a result of CV diseases. The median serum TG/HDL-C ratio was $1.11(\mathrm{IQR}=0.71-1.80)$. In a multivariate Cox regression analysis, patients with higher $\mathrm{TG} / \mathrm{HDL}-\mathrm{C}$ ratio levels (tertile 3 ) had a higher incidence of $\mathrm{CV}$ mortality (adjusted $\mathrm{HR}=2.12 ; 95 \% \mathrm{Cl}: 1.21-3.72 ; P=0.009$ ) and all-cause mortality (adjusted $\mathrm{HR}=2.08 ; 95 \% \mathrm{Cl}: 1.37-3.14 ; P=0.001$ ) compared to patients in tertile 1 . These associations persisted after excluding the patients who have already taken lipid-lowering medications. For older patients (> 60 years), each 1-unit higher baseline TG/HDL-C level was associated with a 48\% (95\% Cl: 1.06-2.07; $P=$ 0.021 ) increased risk of all-cause mortality and a 59\% (95\% Cl: 1.03-2.45; $P=0.038)$ increased risk of CV mortality; however, this association was not observed in patients $\leq 60$ years of age.

Conclusions: A higher serum TG/HDL-C ratio was an independent predictor of all-cause and CV mortality in PD patients. Furthermore, an elevated TG/HDL-C ratio was significantly associated with higher all-cause and CV mortality in older PD patients.

Keywords: Triglyceride/high-density lipoprotein cholesterol (TG/HDL-C) ratio, Mortality, Cardiovascular, Older, Peritoneal dialysis

\section{Introduction}

Peritoneal dialysis (PD) is a primary treatment modality for end-stage renal disease (ESRD) patients [1]. In 2014, 55,373 patients received PD in China [2]. Despite advancements in dialysis treatment technology, ESRD patients continue to experience a lower quality of life, high hospitalization rates, and high annual mortality

\footnotetext{
* Correspondence: chenqinkaiedu@163.com

${ }^{+}$Xiaojiang Zhan and Mei Yang contributed equally to this work.

Department of Nephrology, The First Affiliated Hospital of Nanchang

University, 17\# Yongwai Street, Nanchang 330006, China
}

rates of approximately $20 \%$, a rate worse than that of many cancers [3]. A major cause of mortality is cardiovascular disease (CVD) [2], which is 10-30 fold higher in ESRD patients than in the general population [4]. Traditional CVD risk factors, such as hypercholesterolemia and obesity, have not been able to fully explain the increased mortality observed in ESRD patients. In contrast, some reports in the literature have shown that these factors are associated with better survival in dialysis patients [5-7]. However, uremic dyslipidemia in PD patients, including high levels of triglycerides (TG) and lower

(C) The Author(s). 2019 Open Access This article is distributed under the terms of the Creative Commons Attribution 4.0 International License (http://creativecommons.org/licenses/by/4.0/), which permits unrestricted use, distribution, and 
high-density lipoprotein cholesterol (HDL-C), could lead to an increased risk of CVD-related mortality [8].

Recently, parameters associated with inflammation, oxidative stress, insulin resistance, and endothelial dysfunction have been reported to be better at predicting CVD outcomes [9]. The TG/HDL-C ratio, which is a simple and reproducible parameter that can easily be calculated daily and is known as an atherogenic index of plasma [10], is one such parameter [11]. Accumulated evidence has shown a predictive role for the TG/HDL-C ratio in several disorders, such as fatal and nonfatal cardiovascular (CV) events [10], coronary atherosclerosis $[12,13]$, impaired heart-rate recovery after exercise [14], ischemic heart disease [15], CVD and coronary heart disease [16, 17]. However, dyslipidemia and its association with CVD and mortality in patients on dialysis present a unique challenge in clinical practice, as their effect on outcomes remains to be fully clarified. Recently, a large retrospective study enrolled 50,673 hemodialysis (HD) patients and showed that a higher TG/HDL-C ratio was associated with better CV and overall survival [3]. In contrast, Chen et al. [16] indicated that a higher TG/HDL-C ratio was associated with a higher incidence of $\mathrm{CV}$ and all-cause mortality. The difference between the two studies above may be due to the different types of patients enrolled; the study of Chen et al. [16] enrolled both HD and PD patients.

There is a paucity of data on whether the TG/HDL-C ratio is an independent predictor of mortality in PD patients. Thus far, to the best of our knowledge, only one study has indicated that a higher serum TG/HDL-C ratio is associated with an increased risk of all-cause and CV mortality in PD patients [18]. In contrast, one recent study indicated that the TG/HDL-C ratio was negatively associated with all-cause mortality among elderly adults in the general population [19]. While accumulating evidence supports the predictive power of the TG/HDL-C ratio in general and in certain subgroups, very few studies have focused on the age-related differences between the TG/HDL-C ratio and mortality in PD patients.

The aim of this study was to find an association between the TG/HDL-C ratio and all-cause and CV mortality in PD patients; furthermore, we tried to find age-related differences between the TG/HDL-C ratio and mortality in PD patients.

\section{Methods}

\section{Study population and data source}

The study cohort was comprised of all patients with ESRD who initiated PD between November 1, 2005, and February 28, 2017, in the PD center of The First Affiliated Hospital, Nanchang University, Jiangxi, China. The study enrolled patients who were $\geq 18$ years old, were had been treated with PD therapy only in our PD center for at least 90 days, and had serum TG and HDL-C levels measured during the first 3-months of PD therapy. We excluded patients who were catheterized in other hospitals or transferred from permanent HD or who had experienced failed renal transplantation. Overall, as shown in Fig. 1, a total of 973 patients were enrolled in this study. The TG/HDL-C ratio was treated as a categorical variable and divided into tertiles (Ts): T1 $(\leq 0.833, n=323)$, T2 $(>0.833$ to $\leq 1.496, n=325)$ and T3 $(>1.496, \mathrm{n}=325)$. The reference TG/HDL-C ratio category for all analyses was T1. All study procedures complied with the ethical guidelines of the Declaration of Helsinki and were approved by the Human Ethics Committees. Written informed consent was obtained from all the patients before study enrollment.

All patients were followed up until death, cessation of PD, or May 31, 2017. All baseline data were collected within a 3-month period, starting from the date of first PD therapy. Baseline demographic data included age, sex, primary cause of end-stage renal disease (ESRD), and presence of diabetes and CVD. Clinical and biochemical data included body mass index (BMI), blood pressure, estimated glomerular filtration rate (eGFR), medication use, hemoglobin, white blood cells, serum albumin, serum uric acid, total cholesterol (CHOL), TG, HDL-C and the Charlson comorbidity index (CCI). Baseline residual renal function was assessed by eGFR using the Chronic Kidney Disease Epidemiology Collaboration creatinine equation. The CCI has been used to stratify patients in order to control for the confounding influence of comorbid conditions on overall survival and the score was calculated for each patient as the total of the patient's comorbid conditions which have been weighted. Conditions with a weight of one included: myocardial infarction, congestive heart failure, peripheral vascular disease, cerebrovascular disease, dementia, chronic pulmonary disease, connective tissue disease, ulcer disease, mild liver disease and diabetes. Conditions with a weight of two included: hemiplegia, moderate or severe renal disease, diabetes with end organ damage and any malignancy. Moderate or severe liver disease (e.g., cirrhosis with ascites) was given a weight of 3 and metastatic solid tumor or AIDS received a weight of 6 [20]. The TG/HDL-C ratio was calculated as TG divided by HDL-C (expressed in $\mathrm{mmol} / \mathrm{L}$ ).

\section{Study outcomes}

The primary outcomes were all-cause and CV mortality. Cardiovascular events that contributed to $\mathrm{CV}$ mortality were defined by the first occurrence of myocardial infarction, stroke, heart failure, hospitalization for unstable angina, peripheral vascular event, sudden death, death associated with a cardiovascular procedure, or death due to aneurysm dissection or rupture, fatal pulmonary 


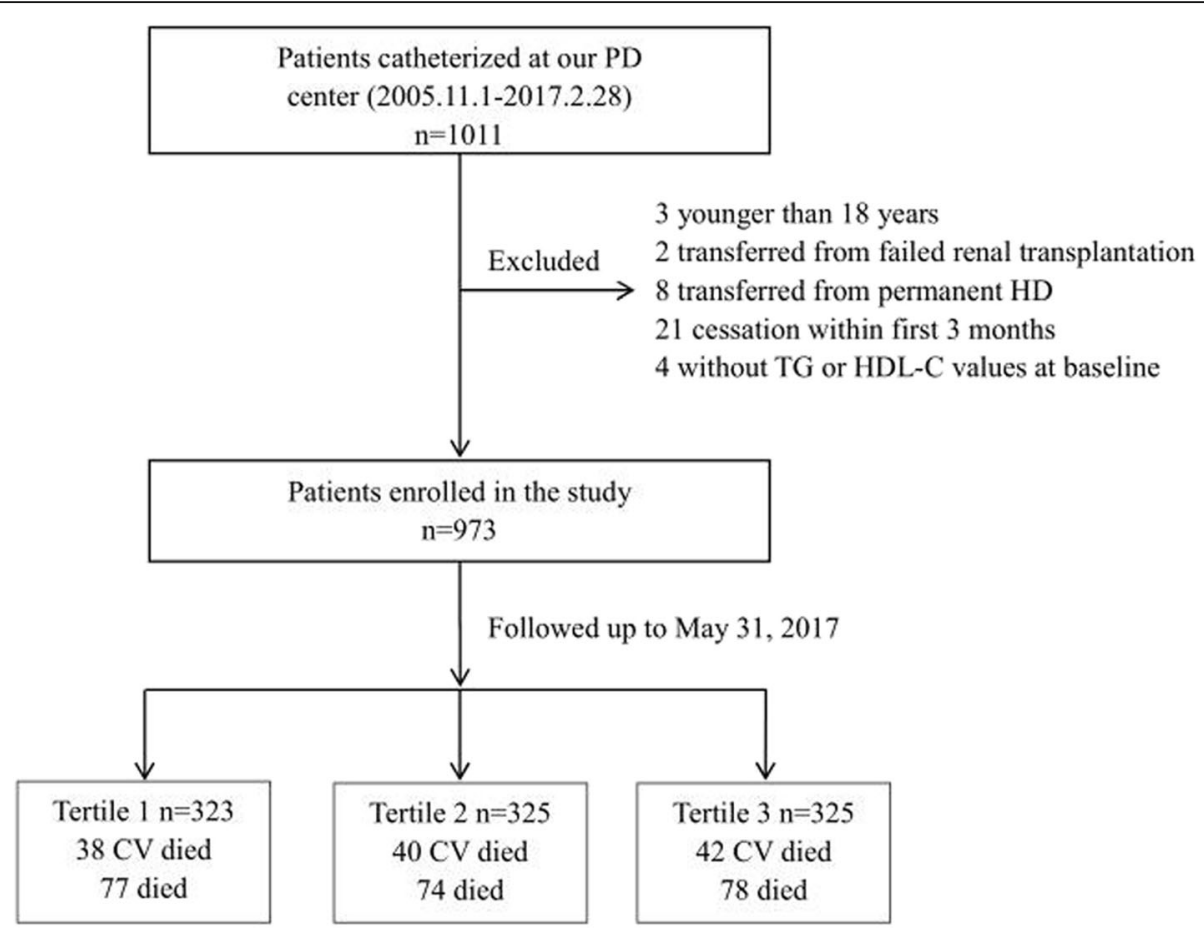

Fig. 1 Flow chart of this study. PD, peritoneal dialysis; HD, hemodialysis; TG, triglyceride; HDL-C, high density lipoprotein cholesterol; $\mathrm{CV}$, cardiovascular

embolism, or death due to other or unknown cardiovascular causes [21].

\section{Statistical analysis}

All statistical analyses were performed using SPSS software version 21.0 (SPSS Inc., Chicago, IL). A $P$ value $<0.05$ was considered to be statistically significant. Continuous variables are presented as the means \pm standard deviations (SDs) or medians and interquartile ranges. Categorical data are reported as frequencies and percentages. The Chi-squared test, Kruskal-Wallis test, and one-way ANOVA were used to compare the baseline characteristics of patients classified by tertile of the TG/ HDL-C ratio. Survival was calculated using the KaplanMeier method, and differences between distributions of survival were assessed by the Breslow test. Multiple Cox proportional regression analysis was used to investigate the independent association of the TG/HDL-C ratio with mortality after adjustment for several confounders. The censored data included switching to HD, renal transplantation, moving to another center, declining additional treatment, loss to follow-up, or still attending our PD center on May 31, 2017. The fully adjusted Cox proportional regression model was adjusted for age, sex, diabetes, history of CVD, hypertension, eGFR, BMI, CCI, white blood cells, hemoglobin, platelet, serum albumin, total cholesterol, uric acid, and use of lipid-lowering agents. The results are presented as hazard ratios
(HRs) $\pm 95 \%$ confidence intervals (CIs), and statistical significance is indicated. In sensitivity analyses, to minimize possible effects of the lipid-lowering agents, all hazard ratios were recalculated after excluding the patients who have already taken lipid-lowering medications.

\section{Results}

\section{Study population}

A total of 973 patients were included in this study. The baseline demographics and clinical and laboratory characteristics of the patients across tertiles of the TG/HDL$\mathrm{C}$ ratio are summarized in Table 1 . The mean age of the patients was $49.67 \pm 14.58$ years, and $57.1 \%$ were male. The percentages of hypertension, diabetes, and CVD history were $73,19.1$, and $9.9 \%$, respectively. Patients with elevated baseline TG/HDL-C ratios compared with the reference group (T1) tended to be younger females who were more likely to have hypertension and diabetes. They also had lower serum HDL-C levels and higher BMIs and total cholesterol and TG levels.

\section{Outcomes according to the TG/HDL-C ratio tertiles}

The median serum TG/HDL-C ratio was 1.11 (IQR = 0.71-1.80). During the median follow-up period of 27.2 months (IQR $=13.4-41.5$ months), 229 (23.5\%) patients died. Of these 229 patients, 184 individuals had data available on the primary cause of death, of which 120 
Table 1 Baseline characteristics of individuals stratified by tertiles of TG/HDL-C ratio

\begin{tabular}{|c|c|c|c|c|c|}
\hline \multirow[t]{2}{*}{ Variables } & \multirow[t]{2}{*}{ Total } & \multicolumn{3}{|l|}{ TG/HDL-C ratio } & \multirow[t]{2}{*}{$P$ value } \\
\hline & & $\begin{array}{l}\mathrm{T} 1 \leq 0.833 \\
(n=323)\end{array}$ & $\begin{array}{l}0.833<\mathrm{T} 2 \leq 1.496 \\
(n=325)\end{array}$ & $\begin{array}{l}\mathrm{T} 3>1.496 \\
(n=325)\end{array}$ & \\
\hline Age (y) & $49.67 \pm 14.58$ & $50.95 \pm 15.09$ & $49.55 \pm 14.18$ & $48.52 \pm 14.41$ & 0.103 \\
\hline Male (\%) & $556(57.1)$ & $198(61.3)$ & $172(52.9)$ & $186(57.2)$ & 0.098 \\
\hline Body mass index (kg/m 2) & $21.91 \pm 3.34$ & $21.17 \pm 2.93$ & $21.83 \pm 3.31$ & $22.73 \pm 3.58$ & $<0.001$ \\
\hline Diabetes (\%) & $186(19.1)$ & $59(18.3)$ & $61(18.8)$ & $66(20.3)$ & 0.789 \\
\hline CVD (\%) & $96(9.9)$ & $28(8.7)$ & $27(8.3)$ & $41(12.6)$ & 0.124 \\
\hline Hypertension (\%) & $710(73)$ & $223(69)$ & $254(78.2)$ & $233(71.7)$ & 0.027 \\
\hline Systolic pressure (mmHg) & $146.91 \pm 25.89$ & $147.48 \pm 25.57$ & $146.96 \pm 24.99$ & $146.28 \pm 27.14$ & 0.84 \\
\hline Diastolic pressure $(\mathrm{mmHg})$ & $87.91 \pm 15.89$ & $87.34 \pm 16.44$ & $88.42 \pm 14.29$ & $87.96 \pm 16.62$ & 0.683 \\
\hline $\mathrm{CCl}$ & $3.53 \pm 1.88$ & $3.60 \pm 1.87$ & $3.48 \pm 1.85$ & $3.51 \pm 1.92$ & 0.711 \\
\hline Total Kt/N & $2.16(1.67,2.70)$ & $2.15(1.51,2.60)$ & $2.20(1.73,2.72)$ & $2.09(1.68,2.74)$ & 0.131 \\
\hline eGFR (ml/min per $1.73 \mathrm{~m} \mathrm{2)}$ & $3.19(1.73,5.50)$ & $2.90(1.69,4.76)$ & $3.16(1.71,5.56)$ & $3.55(1.77,5.82)$ & 0.16 \\
\hline White blood cells (/L) & $5.74(4.50,7.21)$ & $5.33(4.22,6.90)$ & $5.73(4.43,6.95)$ & $6.15(4.97,7.73)$ & $<0.001$ \\
\hline Hemoglobin (g/L) & $78.89 \pm 16.81$ & $76.71 \pm 14.83$ & $78.96 \pm 16.98$ & $80.98 \pm 19.0$ & 0.005 \\
\hline Platelet (/L) & $161.0(118.0,206.5)$ & $151.0(111.0,192.0)$ & $158.0(112.0,206.25)$ & $176.0(129.0,220.25)$ & $<0.001$ \\
\hline Albumin (g/L) & $35.41 \pm 5.25$ & $35.56 \pm 5.06$ & $35.39 \pm 5.41$ & $35.28 \pm 5.28$ & 0.798 \\
\hline uric acid (mmol/L) & $435(347,535)$ & $428.5(342.50,520.75)$ & $441.5(356,540)$ & $439(345,562)$ & 0.314 \\
\hline Total cholesterol (mmol/L) & $4.06(3.37,4.85)$ & $3.99(3.23,4.65)$ & $4.04(3.37,4.82)$ & $4.22(3.49,5.12)$ & 0.005 \\
\hline Triglycerides (mmol/L) & $1.27(0.89,1.76)$ & $0.80(0.63,0.96)$ & $1.26(1.03,1.48)$ & $2.06(1.69,2.57)$ & $<0.001$ \\
\hline $\mathrm{HDL}-\mathrm{C}(\mathrm{mmol} / \mathrm{L})$ & $1.09(0.90,1.39)$ & $1.40(1.18,1.69)$ & $1.09(0.95,1.31)$ & $0.88(0.73,1.03)$ & $<0.001$ \\
\hline Lipid-lowering agents use (\%) & $91(9.4)$ & $23(7.1)$ & $23(7.1)$ & $45(13.8)$ & 0.003 \\
\hline
\end{tabular}

Abbreviations: CVD Cardiovascular disease, CCI Charlson comorbidity index, HDL-C High-density lipoprotein cholesterol, $T$ Tertile $P<0.05$ is considered statistically significant

(65.2\%) deaths were attributed to CV mortality, 19 (10.3\%) to infectious disease, $21(11.4 \%)$ to cachexia, 3 (1.6\%) to malignancy, and $21(9.2 \%)$ to other reasons. The most common cause of CV death in this study was congestive heart failure $(n=69,57.5 \%)$, followed by cerebrovascular accident $(n=42,35 \%)$, unstable angina $(n=$ $4,3.3 \%)$, acute myocardial infarction $(n=3,2.5 \%)$ and cardiac arrhythmia $(n=2,1.7 \%)$.

\section{All-cause and CV mortality}

Kaplan-Meier survival estimates of all-cause and CV mortality with different levels of TG/HDL-C ratios are shown in Fig. 2. Patient survival rates were lower in the T3 group than in the T1 group $(P=0.047)$ (Fig. 2a). Similarly, patients in the T3 group had the lowest CV survival rate among the groups $(P=0.034)$ (Fig. $2 \mathrm{~b})$. The association between the TG/HDL-C ratio and all-cause and CV mortality was determined by using Cox regression analysis. As shown in Table 2, after adjusting for age, sex, diabetes, history of CVD, hypertension, eGFR, BMI, CCI, white blood cells, hemoglobin, platelet, serum albumin, total cholesterol, uric acid and the use of lipid-lowering agents, the TG/HDL-C ratio remained associated with all-cause and CV mortality. In model 4, the HRs and 95\% CIs for tertile
3 versus tertile 1 were: $\mathrm{HR}, 2.08$ (95\% CI: 1.37-3.14; $P=$ 0.001 ) and HR, 2.12 (95\% CI: $1.21-3.72 ; P=0.009)$ for allcause and CV mortality, respectively. In sensitivity analysis, we excluded the patients with lipid-lowering agents used, the results were materially unchanged (data shown in the supplemental file for Table 3). However, when the ratio was examined as a continuous variable, the association between the TG/HDL-C ratio and all-cause and CV mortality was not significant (HR, 1.05; 95\% CI, 0.98-1.13; $P=0.18$, HR, 1.07; 95\% CI, 0.98-1.17; $P=0.113$, for allcause and CV mortality).

Therefore, the association between the TG/HDL-C ratio and all-cause and CV mortality in terms of sex and age was further studied using Cox regression models after adjusting for the covariates mentioned above. As shown in Table 4, with each 1-unit increase in the TG/ HDL-C ratio, the adjusted HRs of all-cause and CV mortality were 1.23 (95\% CI: $1.10-1.37 ; P<0.001)$ and 1.24 (95\% CI: $1.09-1.42-1.41 ; P=0.001$ ), respectively, for female patients; however, the association for male patients was not significant. In the subgroup analysis of age, for older patients ( $>60$ years), each 1 -unit baseline increase in TG/HDL-C was associated with a $48 \%$ (95\% CI: $1.06-$ 2.07; $P=0.021$ ) increased risk of all-cause mortality and 


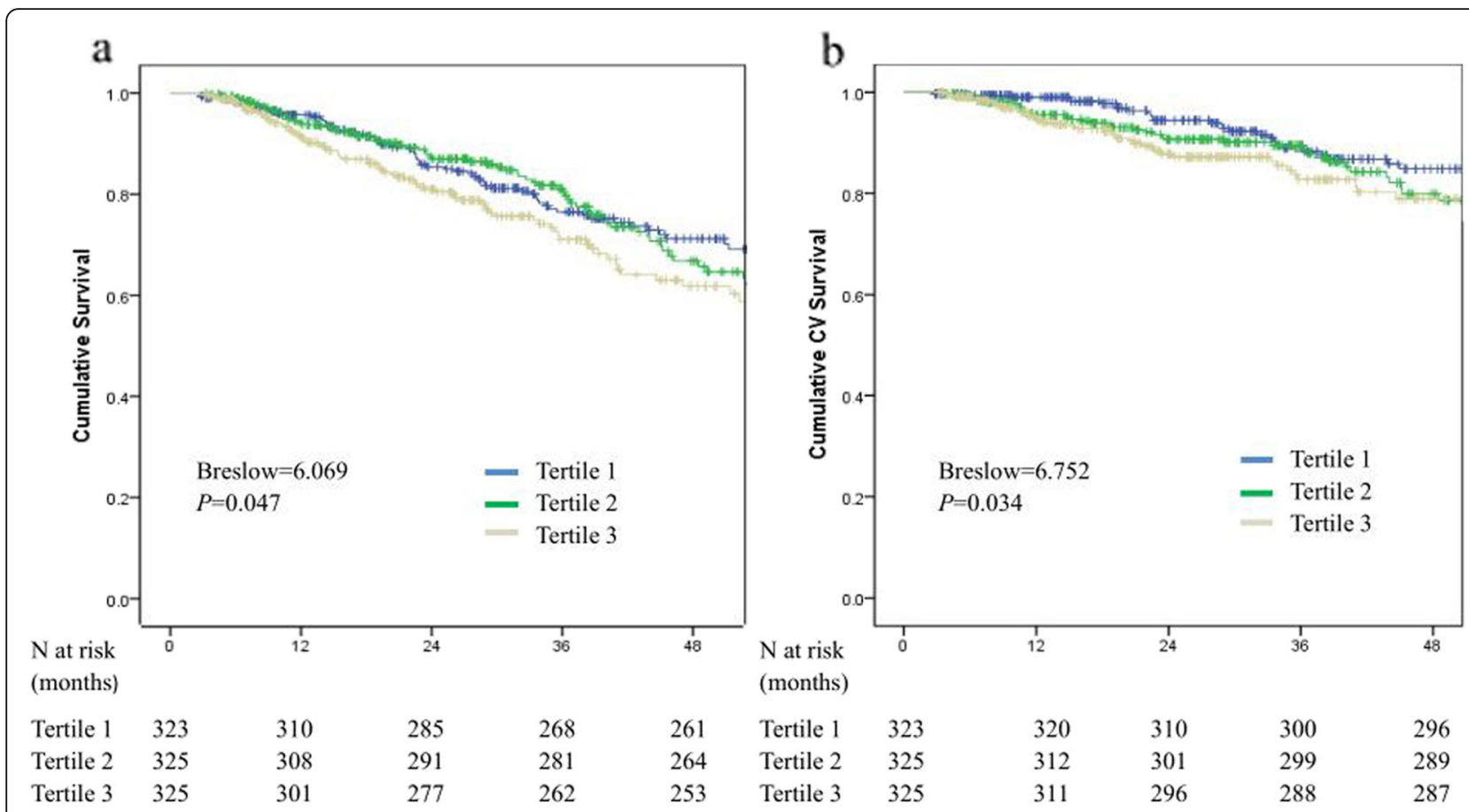

Fig. 2 Kaplan-Meier curves for patients stratified by TG/HDL-C ratio. a All-cause mortality curves. b Cardiovascular mortality curves

a $59 \%$ (95\% CI: $1.03-2.45 ; P=0.038)$ increased risk of $\mathrm{CV}$ mortality. However, these associations were not observed in patients $<60$ years of age.

\section{Discussion}

In this retrospective cohort study, we identified correlations between serum TG/HDL-C ratios and PD patient

Table 2 The associations of TG/HDL-C ratio with all-cause mortality and cardiovascular mortality

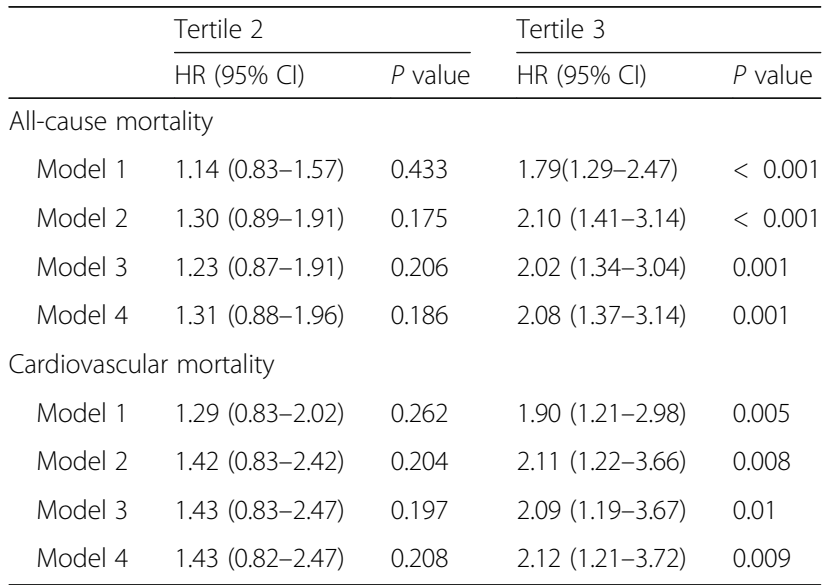

Model 1: adjusted for age, sex

Model 2: model 1 adjusted for diabetes, history of CVD, hypertension, eGFR, $\mathrm{BMI}$, Charlson comorbidity index

Model 3: model 2 adjusted for white blood cells, hemoglobin, platelet, serum albumin, total cholesterol, uric acid

Model 4: model 3 adjusted for lipid-lowering agents use

The tertile 1 as the reference characteristics. Our two principal findings were 1) that higher serum TG/HDL-C ratios independently predicted all-cause and CV mortality in PD patients and the sensitivity analysis indicated the same findings after excluding the patients with the lipid-lowering agents used.2) subgroup analysis demonstrated that an elevated TG/HDL-C ratio was associated with higher allcause and CV mortality in older ( $>60$ years) and female patients.

Table 3 The associations of TG/HDL-C ratio with all-cause and cardiovascular mortality after excluding the patients with the lipid-lowering agents used

\begin{tabular}{|c|c|c|c|c|}
\hline & \multicolumn{2}{|l|}{ Tertile 2} & \multicolumn{2}{|l|}{ Tertile 3} \\
\hline & HR (95\% Cl) & $P$ value & HR $(95 \% \mathrm{Cl})$ & $P$ value \\
\hline \multicolumn{5}{|c|}{ All-cause mortality } \\
\hline Model 1 & $1.11(0.79-1.56)$ & 0.547 & $1.59(1.13-2.23)$ & 0.007 \\
\hline Model 2 & $1.29(0.86-1.94)$ & 0.215 & $1.83(1.20-2.77)$ & 0.005 \\
\hline Model 3 & $1.31(0.87-1.98)$ & 0.201 & $1.72(1012-2.65)$ & 0.013 \\
\hline \multicolumn{5}{|c|}{ Cardiovascular mortality } \\
\hline Model 1 & $1.20(0.74-1.94)$ & 0.454 & $1.61(1.001-2.59)$ & 0.045 \\
\hline Model 2 & $1.32(0.74-2.35)$ & 0.344 & $1.86(1.05-2.35)$ & 0.035 \\
\hline Model 3 & $1.36(0.75-2.44)$ & 0.308 & $1.85(1.02-3.6)$ & 0.043 \\
\hline
\end{tabular}

Model 1: adjusted for age, sex

Model 2: model 1 adjusted for diabetes, history of CVD, hypertension, eGFR, $\mathrm{BMI}$, Charlson comorbidity index

Model 3: model 2 adjusted for white blood cells, hemoglobin, platelet, serum albumin, total cholesterol, uric acid

The tertile 1 as the reference 
Table 4 All-cause mortality and cardiovascular mortality for each 1-unit increase in TG/HDL-C ratio by age and sex

\begin{tabular}{|c|c|c|c|c|c|c|c|c|}
\hline & \multicolumn{2}{|l|}{ Age $\leq 60(y)^{a}$} & \multicolumn{2}{|l|}{ Age $>60(y)^{a}$} & \multicolumn{2}{|l|}{ Male $^{b}$} & \multicolumn{2}{|l|}{ Female $^{b}$} \\
\hline & $\mathrm{HR}(95 \% \mathrm{Cl})$ & $P$ value & $\mathrm{HR}(95 \% \mathrm{Cl})$ & $P$ value & $\mathrm{HR}(95 \% \mathrm{Cl})$ & $P$ value & $\mathrm{HR}(95 \% \mathrm{Cl})$ & $P$ value \\
\hline All-cause mortality & $1.09(0.99-1.18)$ & 0.079 & $1.48(1.06-2.07)$ & 0.021 & $1.04(0.95-1.15)$ & 0.385 & $1.23(1.10-1.37)$ & $<0.001$ \\
\hline Cardiovascular mortality & $1.11(0.99-1.23)$ & 0.061 & $1.59(1.03-2.45)$ & 0.038 & $1.05(0.93-1.19)$ & 0.413 & $1.24(1.09-1.42)$ & 0.001 \\
\hline
\end{tabular}

${ }^{\text {a }}$ Adjusted for sex, diabetes, history of CVD, hypertension, eGFR, BMI, Charlson comorbidity index, white blood cells, hemoglobin, platelet, serum albumin, total cholesterol, uric acid, lipid-lowering agents use

${ }^{\mathrm{b}}$ Adjusted for age, diabetes, history of CVD, hypertension, eGFR, BMI, Charlson comorbidity index, white blood cells, hemoglobin, platelet, serum albumin, total cholesterol, uric acid, lipid-lowering agents use

Our study found that higher serum TG/HDL-C ratios independently predicted all-cause and CV mortality, which are consistent with the previous studies [15, 22]. The mechanisms underlying how a higher TG/HDL-C ratio predicts a higher incidence of all-cause and $\mathrm{CV}$ mortality in PD patients are unclear. There are, however, some potential explanations. First, insulin resistance (IR) may play a role. There are many reports in the literature that discuss how the TG/HDL-C ratio is a useful surrogate for estimating insulin resistance (IR) [23-26]. Moreover, IR is associated with an increased risk of metabolic abnormalities, including hyperglycemia, dyslipidemia, and hypertension [27, 28], which are associated with increases in CV mortality. Importantly, Reardon et al. [29] reported that IR can promote atherogenesis through the IFN $\gamma$-macrophage pathway. Second, the higher TG/ HDL-C ratio represents either increased TG or decreased HDL-C. Currently, evidence suggests that increased TG could play an important role in increased atherosclerosis [30]. However, HDL-C is heterogeneous with anti-atherogenic functions and nonvascular effects [31]. Thus, the TG/HDL-C ratio reflects a balance between atherogenic and protective lipoproteins. Moreover, dyslipidemia, characterized by high levels of TG or low levels of HDL-C, is a common risk factor for atherogenic CVD [32]. Last, oxidative stress and inflammation might be a reason. A previous study showed that the TG/HDL-C ratio was significantly associated with the presence of small and dense low density lipoprotein (LDL) cholesterol particles, which are actively taken up by arterial tissue and cause oxidative damage [33]. Accumulation of oxidized LDL cholesterol stimulates monocytes and macrophages to secrete proinflammatory cytokines and chemokines [34]. Moreover, the different food habit maybe another reason, Scicchitano $\mathrm{P}$ et al. [35] revealed that the food, especially the nutraceuticals, may play a peculiar role in ameliorating human dyslipidemia, and may through their antioxidant action on free radicals or by acting as anti-inflammatory molecules to decrease the incidence and prevalence of cardiovascular events. Therefore, further studies are needed to find the exact mechanisms.
In addition, our study also found that elevated TG/ HDL-C ratio was associated with higher all-cause and $\mathrm{CV}$ mortality in female patients but not in male patients, which are consistent with the previous studies [18, 22]. The mechanism underlying is uncertain; the following may be the causes. It is well know that the TG and HDL cholesterol concentrations vary with gender, moreover, previous study showed that, in the general population, women tend to have lower TG and higher HDL-C levels than their male counterparts, so the women have lower TG/HDL-C ratio than men [36]. Therefore, maybe the dyslipidemia play a pivotal role on higher mortality in female. However, previous studies only indicated the sexrelated difference between the TG/HDL-C ratio and mortality, our study extends previous findings by reporting the age-related difference between the TG/HDL-C ratio and all-cause and $\mathrm{CV}$ mortality. We demonstrated that an elevated TG/HDL-C ratio was associated with higher all-cause and CV mortality in older patients. We could not determine causality or the underlying mechanisms for this phenomenon due to the following potential reasons. As is known to all, throughout the world, the number of people aged 60 years or older has rapidly increased [37]. However, previous studies have indicated that older people have a high prevalence of dyslipidemia, which is an important, modifiable risk factor for CVD [38]. Moreover, low HDL-C levels have already been established as determinants of $\mathrm{CV}$ mortality among elderly populations. This may be due to HDL-C having anti-inflammatory, antioxidant, antiaggregant, anticoagulant and profibrinolytic properties, which promote the maintenance of endothelial functions; thus, the low HDL-C level may accelerate atherogenesis [37, 39].

Our study has several potential limitations that should be considered. First, this was a retrospective study, which can only reveal associations but not causality. Second, these results are from a single center, and the applicability of our findings to other geographical areas is limited. Third, because of the limited sample size, the potential risk factors were not all adjusted for in this cohort study. Hence, the effects of residual confounding factors cannot be completely eliminated. Our future studies will address these issues. 


\section{Conclusions}

In conclusion, our study found that a higher serum TG/ HDL-C ratio was an independent predictor of all-cause and CV mortality in PD patients. Furthermore, the elevated TG/HDL-C ratio was significantly associated with higher all-cause and CV mortality in older PD patients.

\section{Abbreviations}

BMl: Body mass index; CCl: Charlson comorbidity index; CHOL: Total cholesterol; Cls: Confidence intervals; CV: Cardiovascular; CVD: Cardiovascular disease; eGFR: Estimated glomerular filtration rate; ESRD: End-stage renal diseas; HD: Hemodialysis; HDL-C: high-density lipoprotein cholesterol; HR: Hazard ratio; IR: Insulin resistance; LDL: Low density lipoprotein; PD: Peritoneal dialysis; SDs: Standard deviations; TG: Triglycerides; TG/HDL$\mathrm{C}$ : Triglyceride to high-density lipoprotein cholesterol ratio

\section{Acknowledgements}

Not applicable.

\section{Authors' contributions}

XZ conceived the study, RZ, YC, XW and JX participated in the design. XZ performed statistical analyses, MY drafted the manuscript, QC edited and checked the manuscript. All of the authors have read and approved the final manuscript.

\section{Funding}

None.

\section{Availability of data and materials}

All data generated or analyzed during this study are included in this published article.

\section{Ethics approval and consent to participate}

All subjects have given their written informed consent and the study was approved by the ethics committee of The First Affiliated Hospital of Nanchang University. It was conducted according to the standards of the Declaration of Helsinki.

\section{Consent for publication}

Not applicable.

\section{Competing interests}

The authors declare that they have no competing interests.

Received: 7 July 2019 Accepted: 7 November 2019

Published online: 15 November 2019

\section{References}

1. Zhang F, Liu H, Gong X, Liu F, Peng Y, Cheng M, et al. Risk factors for mortality in Chinese patients on continuous ambulatory peritoneal dialysis. Perit Dial Int. 2015:35:199-205.

2. Cai K, Luo Q, Dai Z, Zhu B, Fei J, Xue C, et al. Hypomagnesemia is associated with increased mortality among peritoneal dialysis patients. PLoS One. 2016:11:e0152488

3. Chang TI, Streja E, Soohoo M, Kim TW, Rhee CM, Kovesdy CP, et al. Association of Serum Triglyceride to HDL cholesterol ratio with all-cause and cardiovascular mortality in incident hemodialysis patients. Clin J Am Soc Nephrol. 2017;12:591-602.

4. Sarnak MJ, Levey AS, Schoolwerth AC, Coresh J, Culleton B, Hamm LL, et al. Kidney disease as a risk factor for development of cardiovascular disease: a statement from the American Heart Association councils on kidney in cardiovascular disease, high blood pressure research, clinical cardiology, and epidemiology and prevention. Circulation. 2003;108:2154-69.

5. Lavie CJ, Milani RV, Ventura HO. Obesity and cardiovascular disease: risk factor, paradox, and impact of weight loss. J Am Coll Cardiol. 2009;53: 1925-32.

6. Kalantar-Zadeh K, Kopple JD, Kilpatrick RD, McAllister CJ, Shinaberger CS, Gjertson DW, et al. Association of morbid obesity and weight change over time with cardiovascular survival in hemodialysis population. Am J Kidney Dis. 2005;46:489-500
7. Moradi H, Abhari P, Streja E, Kashyap ML, Shah G, Gillen D, et al. Association of serum lipids with outcomes in Hispanic hemodialysis patients of the west versus east coasts of the United States. Am J Nephrol. 2015;41:284-95.

8. Ikewaki K. In vivo kinetic studies to further understand pathogenesis of abnormal lipoprotein metabolism in chronic kidney disease. Clin Exp Nephrol. 2014;18:261-4.

9. Sonmez A, Yilmaz MI, Saglam M, Unal HU, Gok M, Cetinkaya H, et al. The role of plasma triglyceride/high-density lipoprotein cholesterol ratio to predict cardiovascular outcomes in chronic kidney disease. Lipids Health Dis. 2015;14:29.

10. Turak O, Afsar B, Ozcan F, Oksuz F, Mendi MA, Yayla C, et al. The role of plasma triglyceride/high-density lipoprotein cholesterol ratio to predict new cardiovascular events in essential hypertensive patients. J Clin Hypertens (Greenwich). 2016;18:772-7.

11. Dobiasova M, Frohlich J. The plasma parameter log (TG/HDL-C) as an atherogenic index: correlation with lipoprotein particle size and esterification rate in apoB-lipoprotein-depleted plasma (FER (HDL)). Clin Biochem. 2001;34:583-8.

12. Frohlich J, Dobiasova M. Fractional esterification rate of cholesterol and ratio of triglycerides to $\mathrm{HDL}$-cholesterol are powerful predictors of positive findings on coronary angiography. Clin Chem. 2003:49:1873-80.

13. Drexel H, Aczel S, Marte T, Benzer W, Langer P, Moll W, et al. Is atherosclerosis in diabetes and impaired fasting glucose driven by elevated LDL cholesterol or by decreased HDL cholesterol? Diabetes Care. 2005;28:101-7.

14. Shishehbor MH, Hoogwerf BJ, Lauer MS. Association of triglyceride-to-HDL cholesterol ratio with heart rate recovery. Diabetes Care. 2004;27:936-41.

15. Jeppesen J, Hein HO, Suadicani P, Gyntelberg F. Low triglycerides-high high-density lipoprotein cholesterol and risk of ischemic heart disease. Arch Intern Med. 2001:161:361-6.

16. Chen HY, Tsai WC, Chiu YL, Hsu SP, Pai MF, Yang JY, et al. Triglyceride to high-density lipoprotein cholesterol ratio predicts cardiovascular outcomes in prevalent dialysis patients. Medicine (Baltimore). 2015;94:e619.

17. Abdel-Maksoud MF, Eckel RH, Hamman RF, Hokanson JE. Risk of coronary heart disease is associated with triglycerides and high-density lipoprotein cholesterol in women and non-high-density lipoprotein cholesterol in men. J Clin Lipidol. 2012;6:374-81.

18. Wu H, Xiong L, Xu Q, Wu J, Huang R, Guo Q, et al. Higher serum triglyceride to high-density lipoprotein cholesterol ratio was associated with increased cardiovascular mortality in female patients on peritoneal dialysis. Nutr Metab Cardiovasc Dis. 2015;25:749-55.

19. Shi XM, Lyu YB, Yin ZX, Su LQ, Zhang J, Cai JF, et al. Follow-up study on the effects of lipid ratios on all-cause mortality among elderly adults in longevity areas of China. Zhonghua Yu Fang Yi Xue Za Zhi. 2016;50:594-9.

20. Charlson M, Szatrowski TP, Peterson J, Gold J. Validation of a combined comorbidity index. J Clin Epidemiol. 1994;47:1245-51.

21. Zhan X, Chen Y, Yan C, Liu S, Deng L, Yang Y, et al. Apolipoprotein B/ apolipoprotein $\mathrm{A} 1$ ratio and mortality among incident peritoneal dialysis patients. Lipids Health Dis. 2018:17:117.

22. Bittner V, Johnson BD, Zineh I, Rogers WJ, Vido D, Marroquin OC, et al. The triglyceride/high-density lipoprotein cholesterol ratio predicts all-cause mortality in women with suspected myocardial ischemia: a report from the Women's ischemia syndrome evaluation (WISE). Am Heart J. 2009:157:548-55.

23. Kim JS, Kang HT, Shim JY, Lee HR. The association between the triglyceride to high-density lipoprotein cholesterol ratio with insulin resistance (HOMA-IR) in the general Korean population: based on the National Health and nutrition examination survey in 2007-2009. Diabetes Res Clin Pract. 2012;97:132-8.

24. Hadaegh F, Hatami M, Tohidi M, Sarbakhsh P, Saadat N, Azizi F. Lipid ratios and appropriate cut off values for prediction of diabetes: a cohort of Iranian men and women. Lipids Health Dis. 2010;9:85.

25. Ren X, Chen ZA, Zheng S, Han T, Li Y, Liu W, et al. Association between triglyceride to $\mathrm{HDL}-\mathrm{C}$ ratio (TG/HDL-C) and insulin resistance in Chinese patients with newly diagnosed type 2 diabetes mellitus. PLoS One. 2016;11: e0154345.

26. Young KA, Maturu A, Lorenzo C, Langefeld CD, Wagenknecht LE, Chen $\mathrm{YI}$, et al. The triglyceride to high-density lipoprotein cholesterol (TG/HDL-C) ratio as a predictor of insulin resistance, beta-cell function, and diabetes in Hispanics and African Americans. J Diabetes Complicat. 2019:33:118-22.

27. Bonora E, Kiechl S, Willeit J, Oberhollenzer F, Egger G, Targher G, et al. Prevalence of insulin resistance in metabolic disorders: the Bruneck study. Diabetes. 1998;47:1643-9. 
28. Bonora E, Targher G, Alberiche M, Bonadonna RC, Zenere MB, Saggiani F, et al. Intracellular partition of plasma glucose disposal in hypertensive and normotensive subjects with type 2 diabetes mellitus. J Clin Endocrinol Metab. 2001;86:2073-9.

29. Reardon CA, Lingaraju A, Schoenfelt KQ, Zhou G, Cui C, Jacobs-El H, et al. Obesity and insulin resistance promote atherosclerosis through an IFNgammaregulated macrophage protein network. Cell Rep. 2018;23:3021-30.

30. Nordestgaard BG. Triglyceride-rich lipoproteins and atherosclerotic cardiovascular disease: new insights from epidemiology, genetics, and biology. Circ Res. 2016;118:547-63.

31. Ertek S. High-density lipoprotein (HDL) dysfunction and the future of $\mathrm{HDL}$. Curr Vasc Pharmacol. 2018;16:490-8

32. Wen J, Chen Y, Huang Y, Lu Y, Liu X, Zhou H, et al. Association of the TG/ HDL-C and non-HDL-C/HDL-C ratios with chronic kidney disease in an adult Chinese population. Kidney Blood Press Res. 2017;42:1141-54

33. Kang HT, Shim JY, Lee YJ, Lee JE, Linton JA, Kim JK, et al. Association between the ratio of triglycerides to high-density lipoprotein cholesterol and chronic kidney disease in Korean adults: the 2005 Korean National Health and nutrition examination survey. Kidney Blood Press Res. 2011;34:173-9.

34. Glass CK, Witztum JL. Atherosclerosis. The road ahead. Cell. 2001;104:503-16.

35. Scicchitano P, Cameli M, Maiello M, Modesti PA, Muiesan ML, Novo S, et al. Nutraceuticals and dyslipidaemia: beyond the common therapeutics. $J$ Funct Foods. 2014;6:11-32.

36. Carroll MD, Lacher DA, Sorlie PD, Cleeman Jl, Gordon DJ, Wolz M, et al, Trends in serum lipids and lipoproteins of adults, 1960-2002. JAMA. 2005; 294:1773-81.

37. de Freitas EV, Brandao AA, Pozzan R, Magalhaes ME, Fonseca F, Pizzi O, et al. Importance of high-density lipoprotein-cholesterol (HDL-C) levels to the incidence of cardiovascular disease (CVD) in the elderly. Arch Gerontol Geriatr. 2011;52:217-22.

38. Supiyev A, Nurgozhin T, Zhumadilov Z, Peasey A, Hubacek JA, Bobak M Prevalence, awareness, treatment and control of dyslipidemia in older persons in urban and rural population in the Astana region, Kazakhstan. BMC Public Health. 2017;17:651

39. Corti MC, Guralnik JM, Salive ME, Harris T, Field TS, Wallace RB, et al. HDL cholesterol predicts coronary heart disease mortality in older persons. JAMA. 1995;274:539-44.

\section{Publisher's Note}

Springer Nature remains neutral with regard to jurisdictional claims in published maps and institutional affiliations.

Ready to submit your research? Choose BMC and benefit from:

- fast, convenient online submission

- thorough peer review by experienced researchers in your field

- rapid publication on acceptance

- support for research data, including large and complex data types

- gold Open Access which fosters wider collaboration and increased citations

- maximum visibility for your research: over $100 \mathrm{M}$ website views per year

At $\mathrm{BMC}$, research is always in progress.

Learn more biomedcentral.com/submissions 